\title{
Discovery sulfoglycomics and identification of the characteristic fragment ions for high sensitivity precise mapping of adult zebrafish brain-specific glycotopes
}

Huan-Chuan Tseng1,2, Cheng-Te Hsiao¹, Nao Yamakawa3, Yann Guérardel4,5, Kay-Hooi Khoo1,2,*

${ }^{1}$ Institute of Biological Chemistry, Academia Sinica, Taiwan

2 Institute of Biochemical Sciences, National Taiwan University, Taiwan;

3 University Lille, CNRS, INSERM, CHU Lille, Institut Pasteur de Lille, US 41-UMS 2014-PLBS, Lille, France.

4 Université de Lille, CNRS, UMR 8576 - UGSF - Unité de Glycobiologie Structurale et Fonctionnelle, F- 59000 Lille, France.,

${ }^{5}$ Institute for Glyco-core Research (iGCORE), Gifu University, Gifu, Japan.

*Correspondence to: Kay-Hooi Khoo, Email: kkhoo@gate.sinica.edu.tw

\section{Supplementary Material}

1. Supplemental Figures S1-S3 (this file)

Fig S1. MALDI-MS profiles of permethylated non-sulfated N-glycans from brain, intestine and ovary.

Fig S2. MALDI-MS profiles of permethylated non-sulfated O-glycans from brain, intestine and ovary.

Fig S3. Individual extracted ion chromatogram plots of major non-sulfated N-glycans from brain, intestine and ovary.

Fig S4. Annotated HCD MS² spectra of the identified major non-sulfated N-glycans.

Fig S5. Annotated negative mode HCD MS2 spectra of the identified HNK-1 carrying hybrid type $\mathrm{N}$-glycans from brain.

Fig S6. Annotated negative mode HCD MS² spectra of the identified Man-6-phosphate carrying Nglycans from brain and intestine.

2. Supplemental Tables S1-S6 (available separately as Excel file)

Listing of productive HCD-MS ${ }^{2}$ scans acquired on the permethylated non-sulfated N- and Oglycans from brain, intestine and ovary, with precursor ions that can be fitted to reasonable glycosyl compositions.

\section{Supplemental Tables S7-S10}

Listing of permethylated sulfated $\mathrm{N}$ - and $\mathrm{O}$-glycans identified from brain and intestine, with their expected versus detected $m / z$ values and assigned glycosyl compositions. 


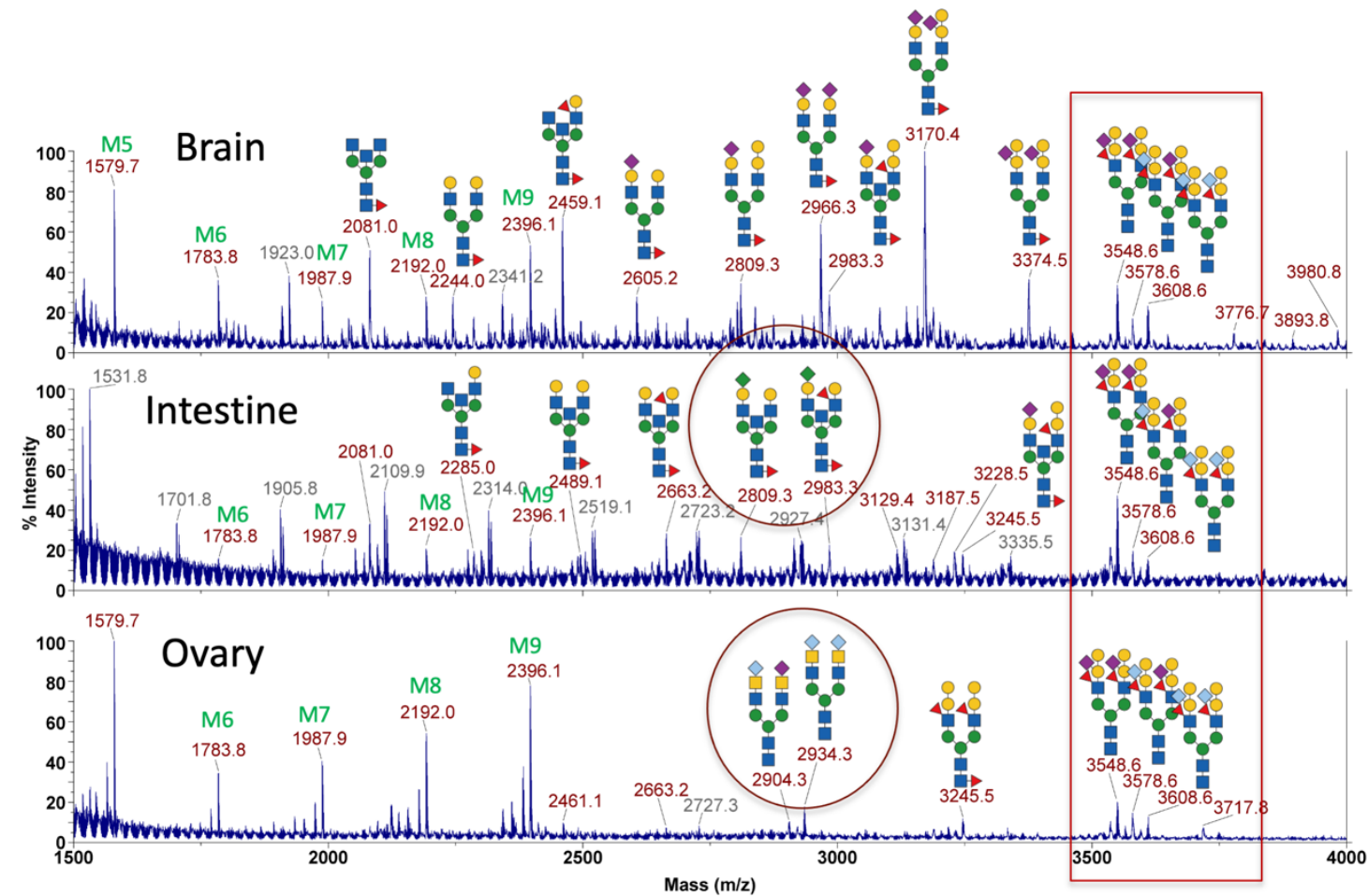

Figure S1. MALDI-MS profiles of permethylated non-sulfated N-glycans from brain, intestine and ovary. Major glycans were assigned based on glycosyl composition and subsequent LC-MS/MS data. M5-M9 denote high mannose N-glycans, Man ${ }_{5} \mathrm{GlcNAc}_{2}-\mathrm{Man}_{9} \mathrm{GlcNAc}_{2}$. The N-glycans circled are unique to intestine and ovary. The $\mathrm{N}$-glycans boxed are common to all three organs.
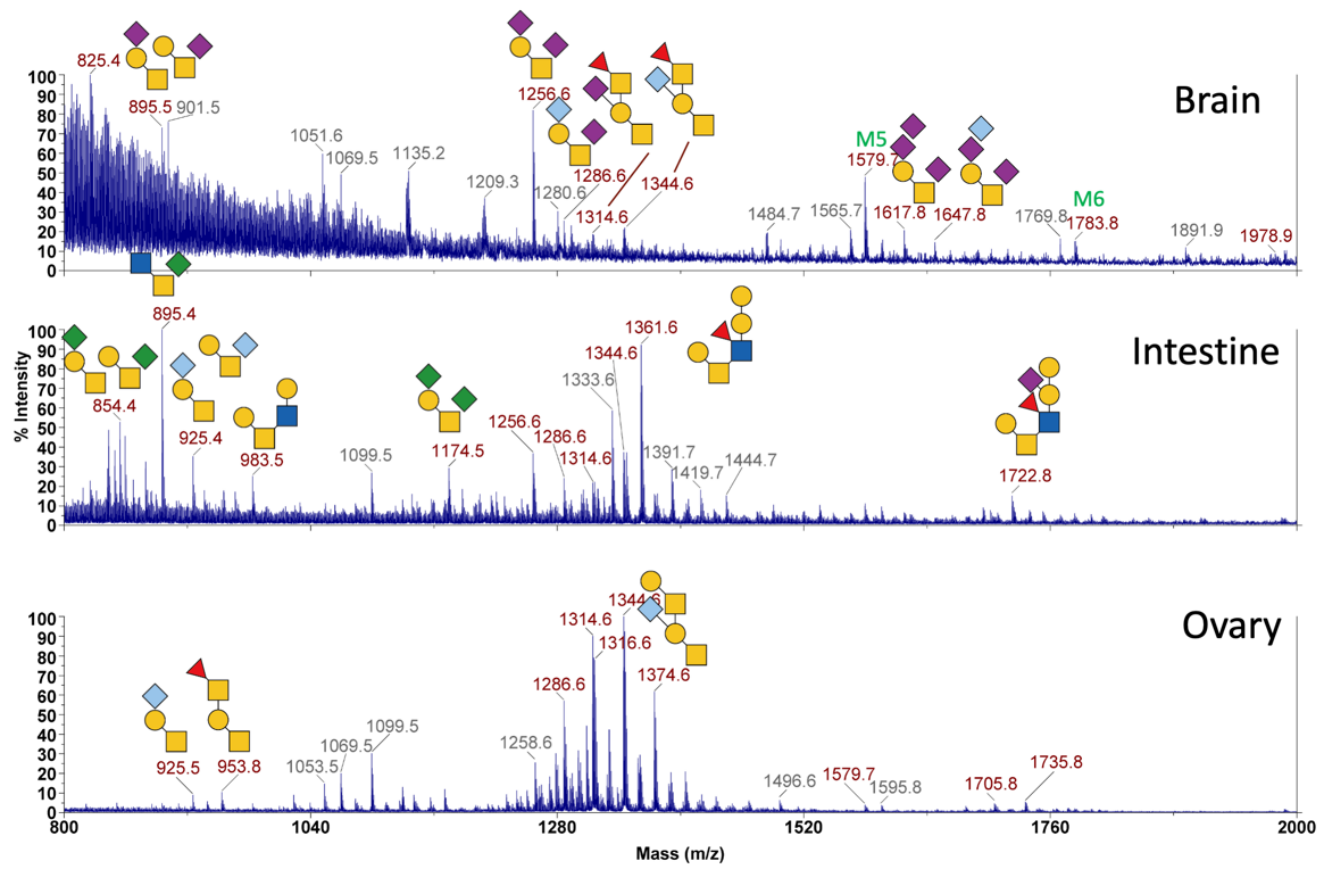

Figure S2. MALDI-MS profiles of permethylated non-sulfated 0-glycans from brain, intestine and ovary. Major glycans were assigned based on glycosyl composition and subsequent LC-MS/MS data. Unassigned or contaminant peaks are labeled by its $m / z$ values in grey. 

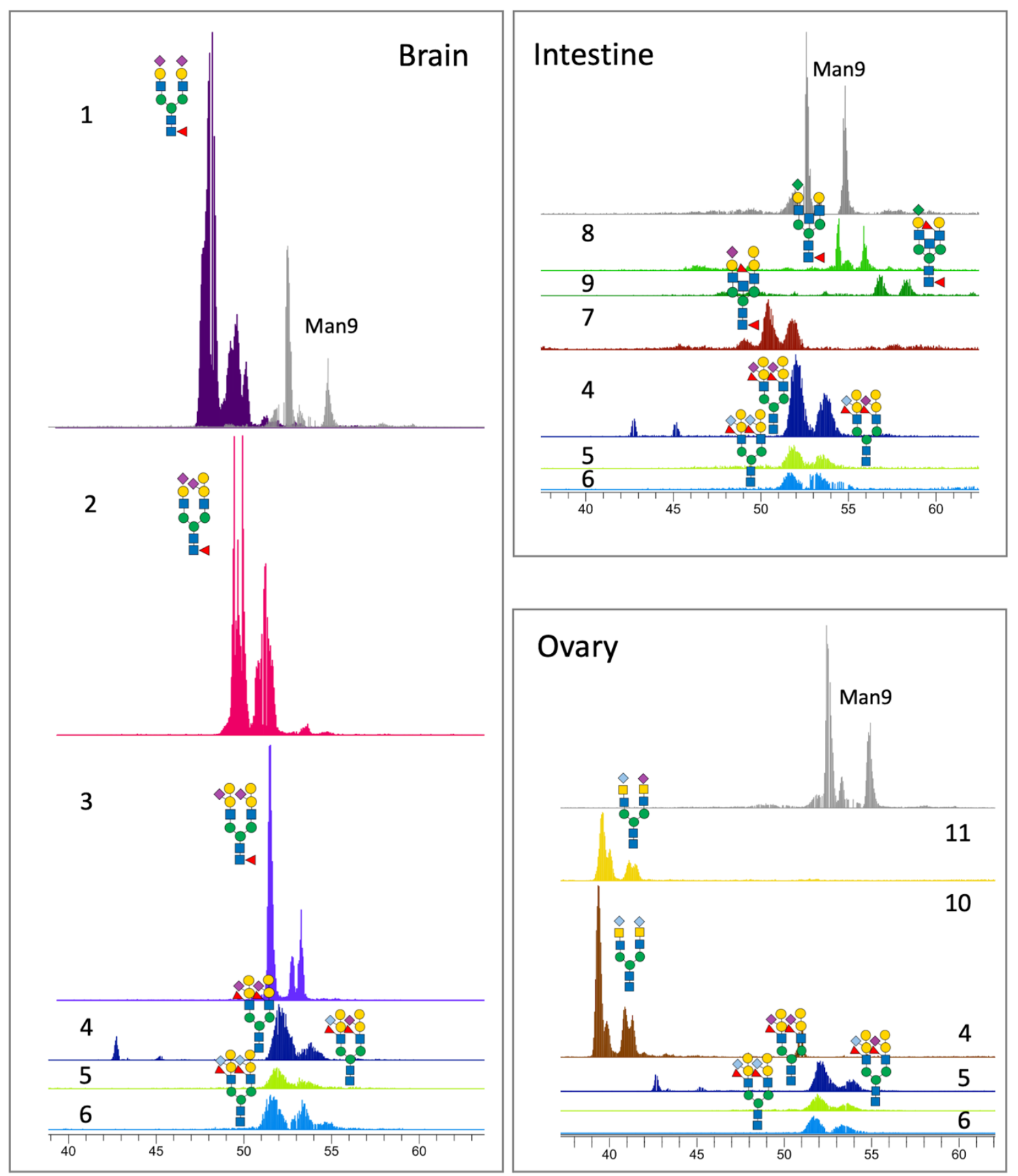

Fig S3. Individual extracted ion chromatogram plots of major non-sulfated $\mathrm{N}$-glycans from brain, intestine and ovary. The same overlaid plots similarly normalized to that of the commonly found $\mathrm{Man}_{9} \mathrm{GlcNAc}_{2}$ (M9) structure in each of the three organs are presented in Fig. 1A and the 11 distinct major complex type structures identified are similarly named 1-11 here. Due to non-reduced reducing end, each was separated into at least 2 peaks by their reducing end anomeric configurations. Additional isomeric structures contributed to extra peaks, which were not individually delineated. Note that structures 4, 5 and 6 are commonly found in all three organs at comparable level relative to M9, with least amount found in ovary. Additional information can be found in the legend to Fig. 1. 
1. $[\mathrm{M}+3 \mathrm{H}]^{3+}=982.5$
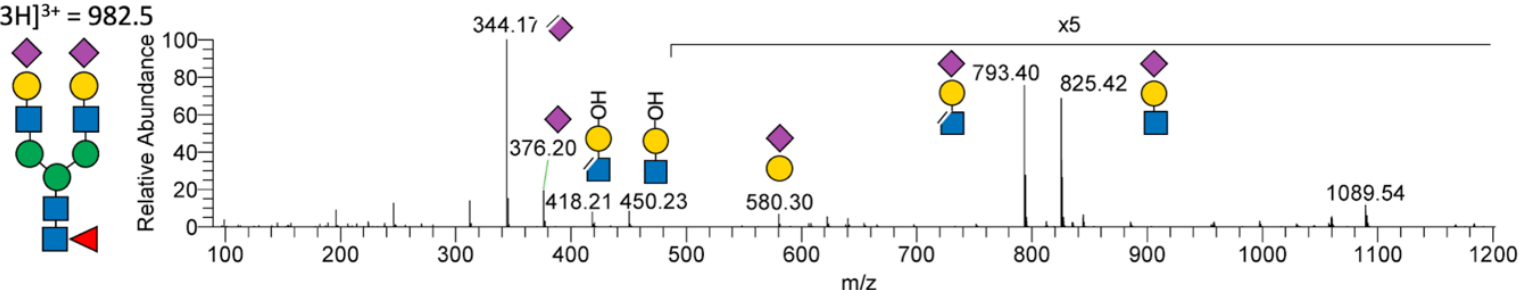

2. $[\mathrm{M}+3 \mathrm{H}]^{3+}=1050.5$
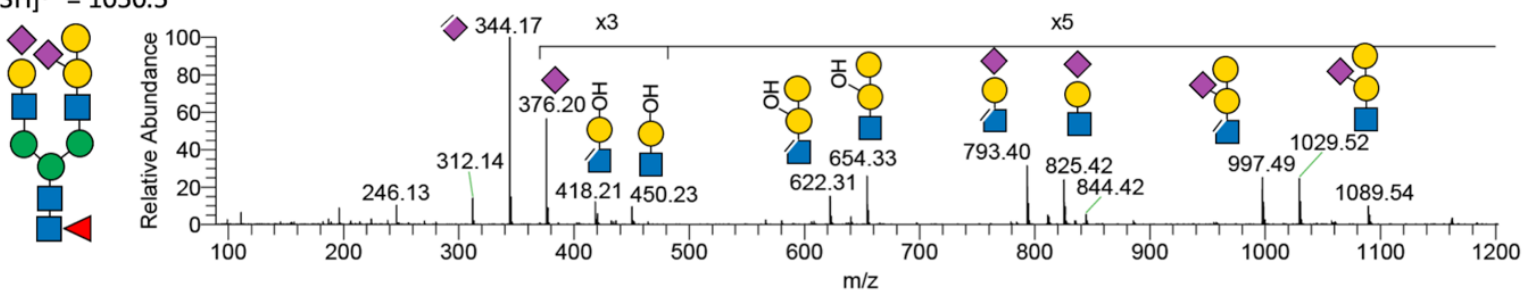

3. $[\mathrm{M}+3 \mathrm{H}]^{3+}=1118.6$
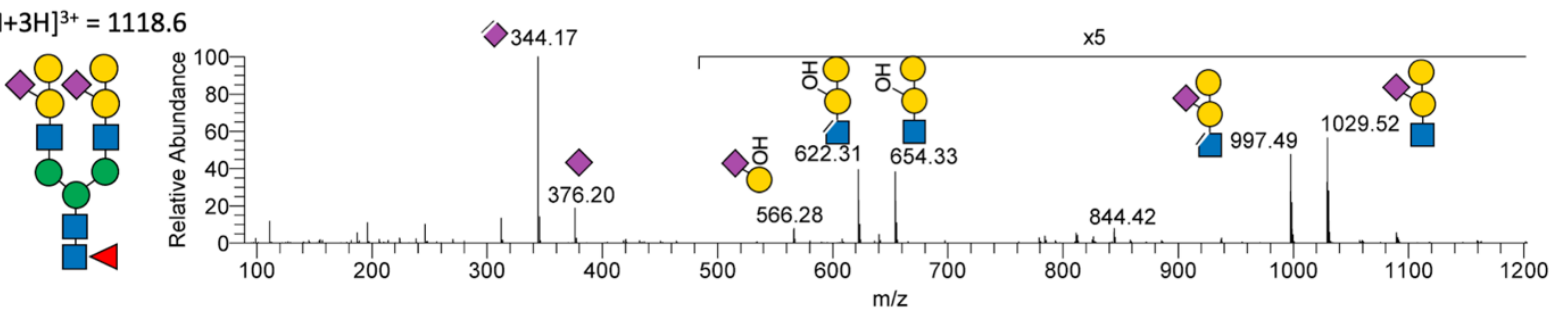

4. $[\mathrm{M}+3 \mathrm{H}]^{3+}=1176.5$
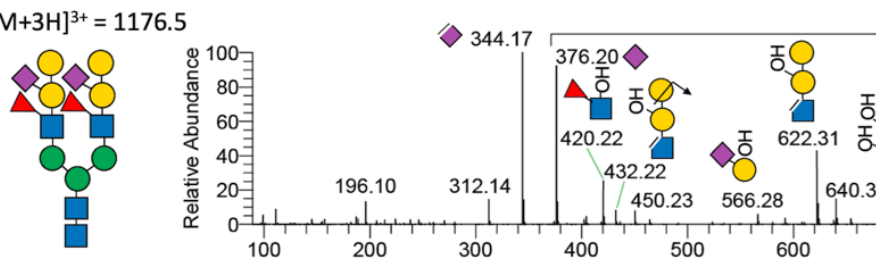

$\mathrm{x} 5$
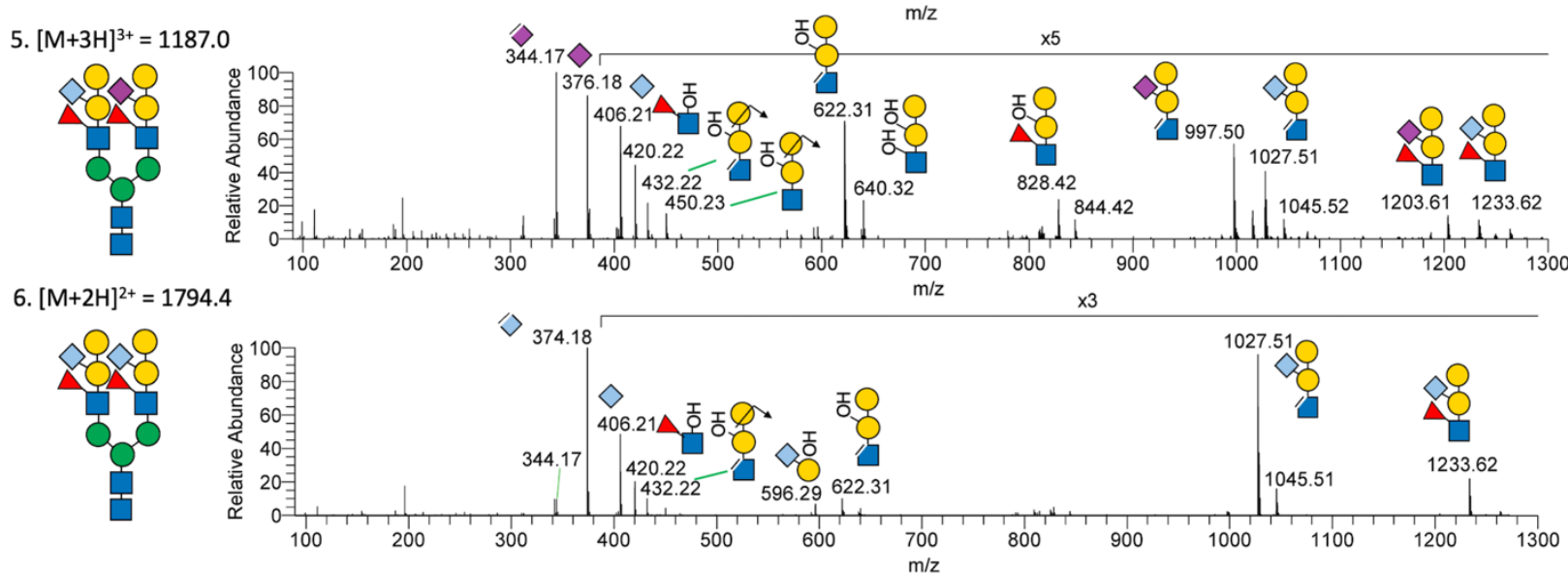

Fig S4. Annotated HCD MS ${ }^{2}$ spectra of the identified major non-sulfated N-glycans. The best spectra for each of the unique N-glycans identified are shown. It should be noted that, in some cases, the MS2 ions could be contributed by more than one co-eluting, co-isolated isomeric or isobaric parent structures within the precursor isolation window width. The deduced N-glycan structures 111 (shown here and in Fig 1) are simply the best fit structures consistent with most, if not all, of the fragment ions. Continue to next page for structures 7-11. 
7. $[\mathrm{M}+3 \mathrm{H}]^{3+}=1069.9$
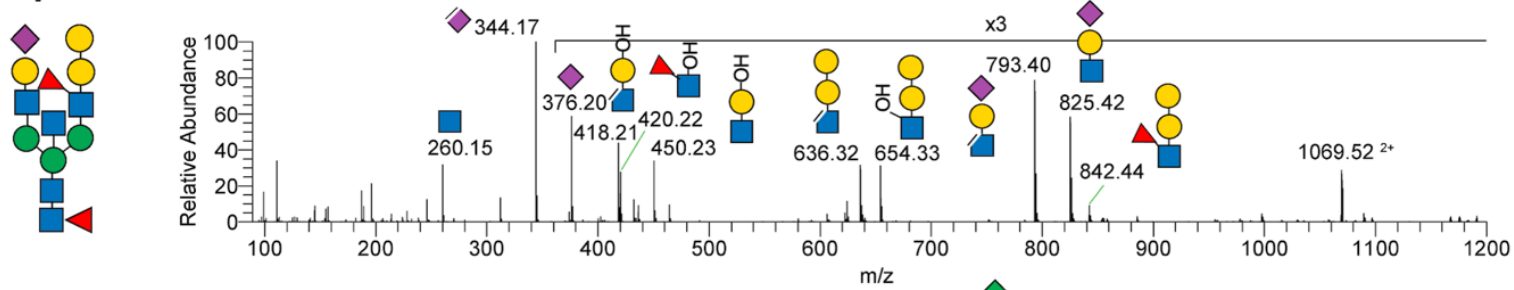

8. $[\mathrm{M}+2 \mathrm{H}]^{2+}=1394.7$
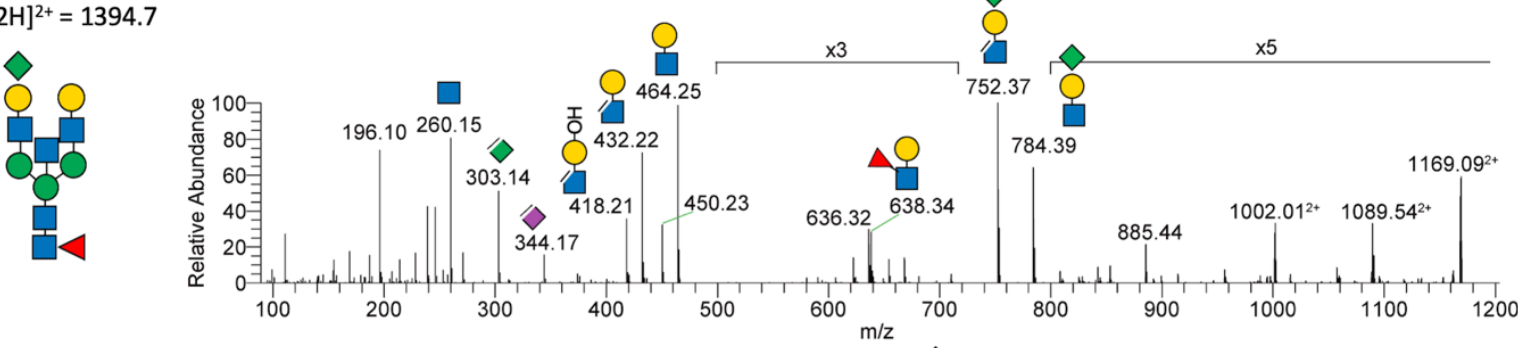

9. $[\mathrm{M}+2 \mathrm{H}]^{2+}=1481.8$
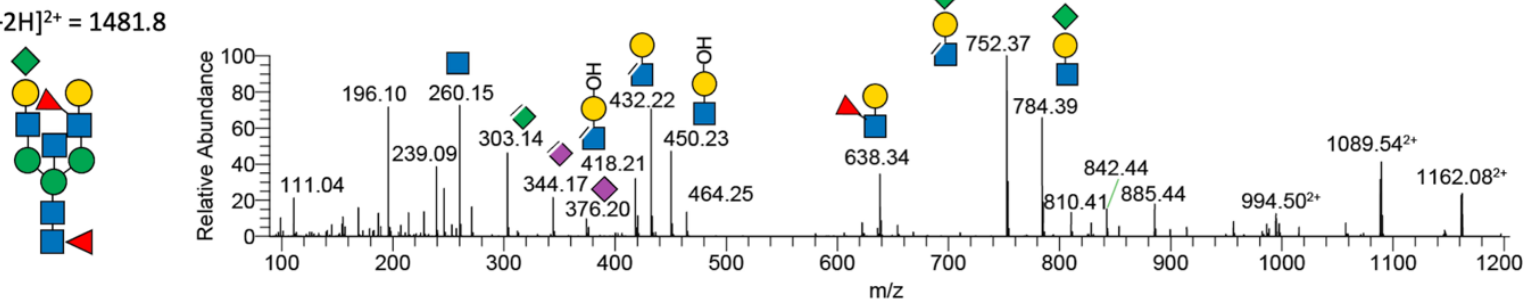

10. $[\mathrm{M}+2 \mathrm{H}]^{2+}=1442.2$
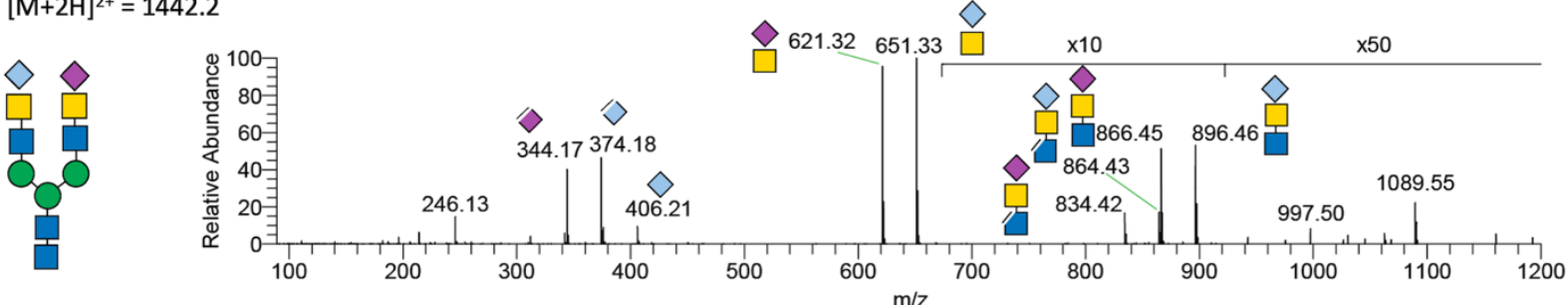

11. $[\mathrm{M}+2 \mathrm{H}]^{2+}=1457.2$
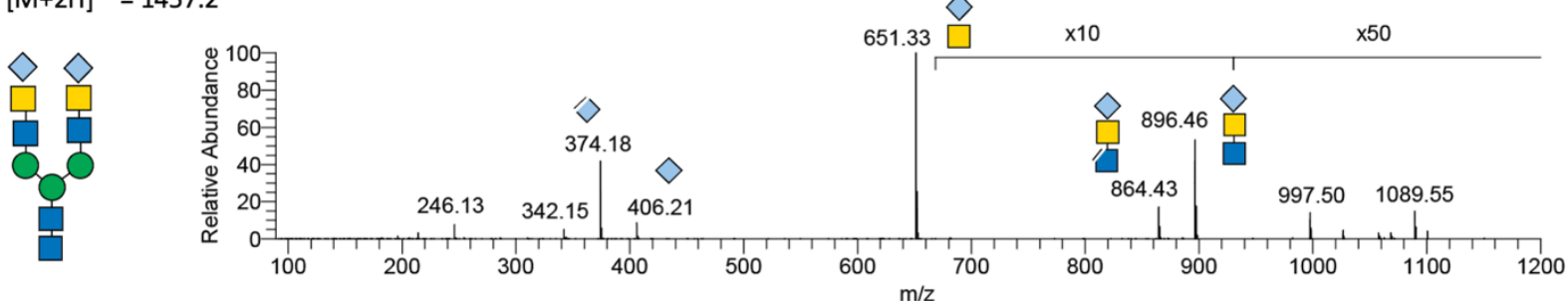

Fig S4. Annotated HCD MS ${ }^{2}$ spectra of the identified major non-sulfated N-glycans. Continued from previous page, showing here the MS² spectra for structures 7-11. 

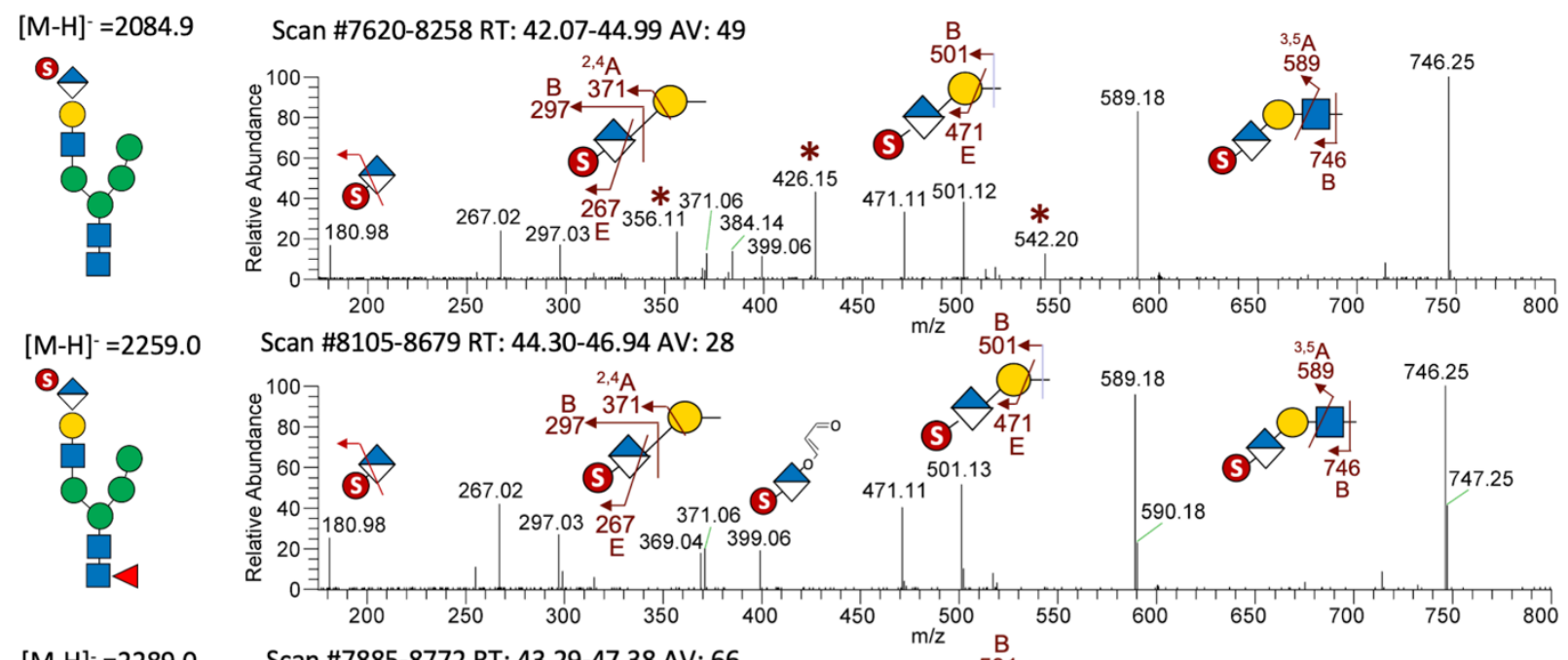

$[\mathrm{M}-\mathrm{H}]^{-}=2289.0$
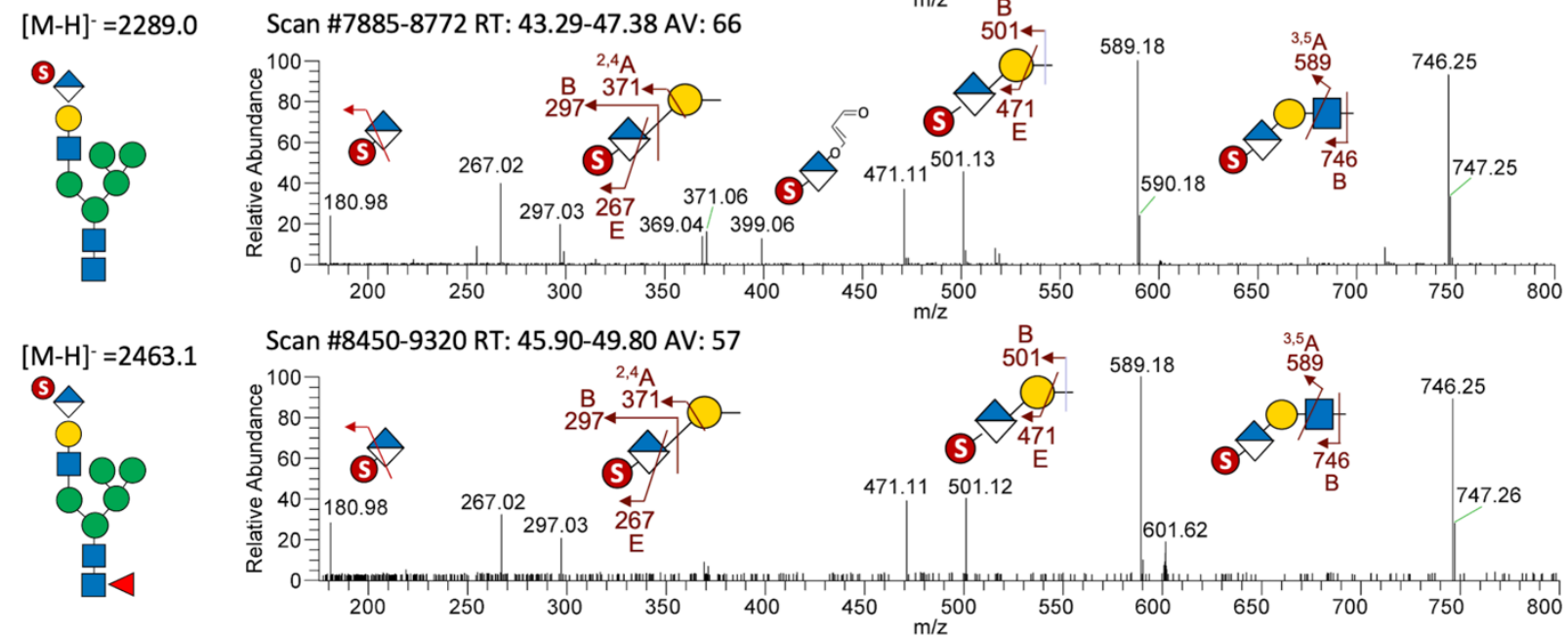

Fig S5. Annotated negative mode HCD MS 2 spectra of the identified HNK-1 carrying hybrid type N-glycans from brain. LC-MS2 spectra were acquired on the singly charged [M-H]- precursors, with their $m / z$ values and deduced structures shown in the left panel. Only the low mass region $(\mathrm{m} / \mathrm{z}<800)$ contained useful signals including the few diagnostic ions of the 3-0-sulfated HNK-1 glycotope, as annotated. The MS2 spectrum for the doubly charged complex type N-glycans carrying 2 HNK-1 glycotopes is shown in Fig. 3F(iv). The hybrid type structure with 1 or 2 Man on the other arm of the trimannosyl core and the core fucosylation were deduced based on absence of other terminal sulfated glycotopes and any extra HexNAc. Note that the MS2 ions produced by the precursors at $m / z$ 2084 and 2289 from brain as shown here are very different from those produced by the isobaric Man-6-phosphate-GlcNAc containing precursors of similar $m / z$ values from intestine (shown in Fig. S6). However, the precursor at $m / z 2084$ from brain also afforded diagnostic ions of Man-6phosphate-GlcNAc $\left(^{*}\right)$, indicative of its presence alongside the HNK-1 carrying isobaric structure. 


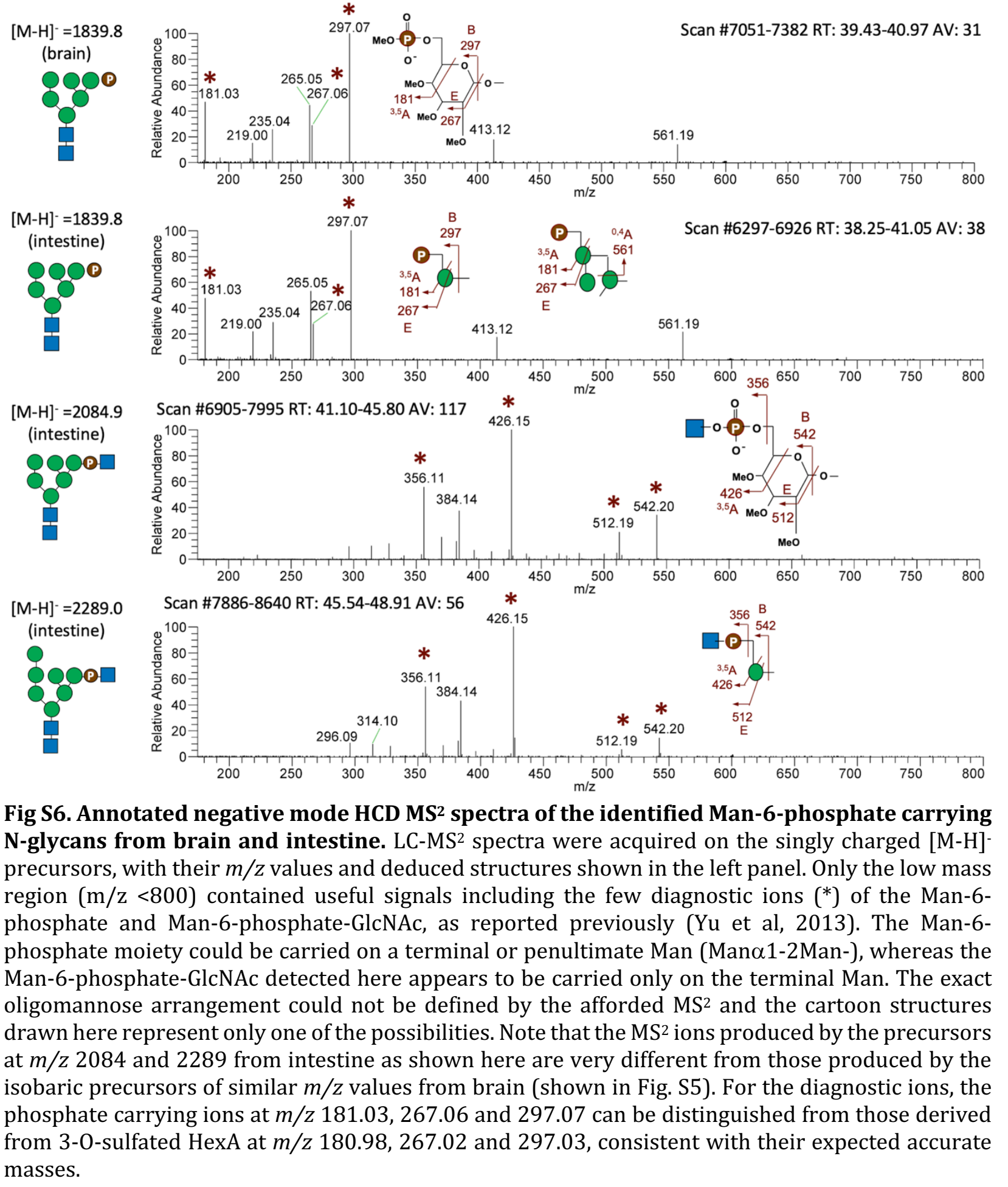

Ref: Yu, S.Y., Chang, L.Y., Cheng, C.W., Chou, C.C., Fukuda, M.N. and Khoo, K.H. (2013) Priming mass spectrometry-based sulfoglycomic mapping for identification of terminal sulfated lacdiNAc glycotope. Glycoconj J. 30, 183-194. doi:10.1007/s10719-012-9396-z 
Table S7. LC-MS data for assigned adult zebrafish brain monosulfated N-glycans.

\begin{tabular}{|c|c|c|c|c|c|c|c|c|c|c|c|c|c|}
\hline \multirow{2}{*}{ RT } & \multicolumn{3}{|c|}{ Monoisotopic $\mathrm{m} / \mathrm{z}$ for $[\mathrm{M}-\mathrm{H}]-$} & \multirow{2}{*}{$\begin{array}{c}\text { Intensity } \\
\text { (XIC, LC-MS) }\end{array}$} & \multirow{2}{*}{ Z } & \multicolumn{8}{|c|}{ Assigned Glycosyl Composition + Sulfate/Phosphate } \\
\hline & Theorectical & MALDI MS & LC-MS & & & $\mathrm{dHex}$ & Hex & HexNAc & NeuAc & NeuGc & GIcA & Sulfate & Phosphate \\
\hline 40.13 & 1839.8426 & 1839.7241 & 1839.8521 & 105872673 & -1 & 0 & 6 & 2 & 0 & 0 & 0 & 0 & 1 \\
\hline 42.17 & 2084.9326 & 2084.8076 & 2084.9309 & 88534741 & -1 & 0 & 5 & 3 & 0 & 0 & 1 & 1 & 0 \\
\hline 44.49 & 2259.0218 & 2258.8562 & 2259.0127 & 477102458 & -1 & 1 & 5 & 3 & 0 & 0 & 1 & 1 & 0 \\
\hline 44.49 & 2289.0324 & 2288.8662 & 2289.0217 & 540221756 & -1 & 0 & 6 & 3 & 0 & 0 & 1 & 1 & 0 \\
\hline 46.77 & 2463.1216 & 2462.9346 & 2463.1135 & 144236041 & -1 & 1 & 6 & 3 & 0 & 0 & 1 & 1 & 0 \\
\hline 45.81 & 2647.2417 & 2647.0168 & 2647.2354 & 37108766 & -1 & 1 & 5 & 4 & 1 & 0 & 0 & 1 & 0 \\
\hline 49.36 & 2865.3218 & 2865.0823 & 2865.3113 & 33549925 & -1 & 1 & 5 & 4 & 1 & 0 & 1 & 1 & 0 \\
\hline 49.76 & 3008.4154 & 3008.1533 & 3008.4070 & 62337070 & -1 & 1 & 5 & 4 & 2 & 0 & 0 & 1 & 0 \\
\hline 51.69 & 3212.5162 & 3212.2246 & 3212.4978 & 66815243 & -1 & 1 & 6 & 4 & 2 & 0 & 0 & 1 & 0 \\
\hline 53.60 & 3416.6159 & 3416.3044 & 3416.6008 & 7446029 & -1 & 1 & 7 & 4 & 2 & 0 & 0 & 1 & 0 \\
\hline
\end{tabular}

Table S8. LC-MS data for assigned adult zebrafish intestine monosulfated $\mathbf{N}$-glycans.

\begin{tabular}{|c|c|c|c|c|c|c|c|c|c|c|c|c|c|}
\hline \multirow{2}{*}{ RT } & \multicolumn{3}{|c|}{ Monoisotopic m/z for [M-H]- } & \multirow{2}{*}{$\begin{array}{c}\text { Intensity } \\
\text { (XIC LC-MS) }\end{array}$} & \multirow{2}{*}{ Z } & \multicolumn{8}{|c|}{ Assigned Glycosyl Composition + Sulfate/Phosphate } \\
\hline & Theorectical & MALDI MS & LCMS & & & $\mathrm{dHex}$ & Hex & HexNAc & NeuAc & NeuGc & KDN & Sulfate & Phosphate \\
\hline 38.43 & 1839.8426 & 1839.7181 & 1839.8479 & 121745580 & -1 & 0 & 6 & 2 & 0 & 0 & 0 & 0 & 1 \\
\hline 42.97 & 2084.9689 & 2084.8230 & 2084.9783 & 646029806 & -1 & 0 & 6 & 3 & 0 & 0 & 0 & 0 & 1 \\
\hline 45.87 & 2289.0687 & 2288.8997 & 2289.0745 & 180907290 & -1 & 0 & 7 & 3 & 0 & 0 & 0 & 0 & 1 \\
\hline 42.26 & 2327.0956 & 2326.8850 & 2327.0898 & 59661814 & -1 & 1 & 4 & 5 & 0 & 0 & 0 & 1 & 0 \\
\hline 39.52 & 2357.1062 & 2356.8940 & 2357.1042 & 24523984 & -1 & 0 & 5 & 5 & 0 & 0 & 0 & 1 & 0 \\
\hline 43.84 & 2531.1954 & 2530.9646 & 2531.1899 & 56651108 & -1 & 1 & 5 & 5 & 0 & 0 & 0 & 1 & 0 \\
\hline 51.20 & 2677.2533 & 2677.0005 & 2677.2456 & 21974525 & -1 & 0 & 5 & 5 & 0 & 0 & 1 & 1 & 0 \\
\hline 52.86 & 2851.3425 & 2851.0693 & 2851.3291 & 152171693 & -1 & 1 & 5 & 5 & 0 & 0 & 1 & 1 & 0 \\
\hline 53.92 & 3025.4317 & 3025.1370 & 3025.4197 & 15917331 & -1 & 2 & 5 & 5 & 0 & 0 & 1 & 1 & 0 \\
\hline
\end{tabular}

Table S9. LC-MS data for assigned adult zebrafish brain disulfated $\mathrm{N}$-glycans.

\begin{tabular}{|c|c|c|c|c|c|c|c|c|c|c|c|c|}
\hline \multirow{2}{*}{ RT } & \multicolumn{2}{|c|}{ Theorectical monoisotopic $\mathrm{m} / \mathrm{z}$} & \multirow{2}{*}{$\begin{array}{l}\text { Observed } \mathrm{m} / \mathrm{z} \\
\text { by LC-MS }\end{array}$} & \multirow{2}{*}{$\begin{array}{c}\text { Intensity } \\
\text { (XIC LC-MS) }\end{array}$} & \multirow{2}{*}{ Z } & \multicolumn{7}{|c|}{ Assigned Glycosyl Composition + Sulfate } \\
\hline & {$[\mathrm{M}-\mathrm{H}]-$} & {$[\mathrm{M}-2 \mathrm{H}] 2-$} & & & & $\mathrm{dHex}$ & Hex & HexNAc & NeuAc & NeuGc & GIcA & Sulfate \\
\hline 31.29 & 2409.0661 & 1224.5437 & 1224.5452 & 29753726 & -2 & 1 & 3 & 6 & 0 & 0 & 0 & 2 \\
\hline 33.66 & 2613.1659 & 1306.0790 & 1306.083 & 11329525 & -2 & 1 & 5 & 5 & 0 & 0 & 0 & 2 \\
\hline 35.04 & 2729.2153 & 1364.1040 & 1364.1052 & 15842492 & -2 & 1 & 5 & 4 & 1 & 0 & 0 & 2 \\
\hline 39.90 & 2804.1997 & 1401.5962 & 1401.5967 & 20469764 & -2 & 1 & 5 & 4 & 0 & 0 & 2 & 2 \\
\hline 37.37 & 2947.2943 & 1473.1435 & 1473.1456 & 12324940 & -2 & 1 & 5 & 4 & 1 & 0 & 1 & 2 \\
\hline 37.42 & 3090.3889 & 1544.6908 & 1544.6927 & 26484022 & -2 & 1 & 5 & 4 & 2 & 0 & 0 & 2 \\
\hline 42.50 & 3294.4887 & 1646.7407 & 1646.7404 & 14829109 & -2 & 1 & 6 & 4 & 2 & 0 & 0 & 2 \\
\hline 46.55 & 3498.5864 & 1748.7906 & 1748.7921 & 6006253 & -2 & 1 & 7 & 4 & 2 & 0 & 0 & 2 \\
\hline
\end{tabular}

Table S10 LC-MS data for assigned adult zebrafish monosulfated O-glycans.

\begin{tabular}{|c|c|c|c|c|c|c|c|c|c|c|c|c|c|}
\hline \multirow{2}{*}{ RT } & \multicolumn{3}{|c|}{ Monoisotopic m/z for [M-H]- } & \multirow{2}{*}{$\begin{array}{c}\text { Intensity } \\
\text { (XIC, LCMS) }\end{array}$} & \multirow{2}{*}{ Z } & \multicolumn{7}{|c|}{ Assigned Glycosyl Composition + Sulfate } & \multirow{2}{*}{ Source } \\
\hline & Theorectical & MALDI MS & LC-MS & & & $\mathrm{dHex}$ & Hex & HexNAc & NeuAc & NeuGc & KDN & Sulfate & \\
\hline 25.07 & 937.4067 & 937.4319 & 937.4066 & 38853547 & -1 & 0 & 1 & 1 & 1 & 0 & 0 & 1 & Brain \\
\hline 28.96 & 896.3802 & 896.3118 & 896.3859 & 90235197 & -1 & 0 & 1 & 1 & 0 & 0 & 1 & 1 & Intestine \\
\hline 24.51 & 937.4067 & 937.3429 & 937.4173 & 491478704 & -1 & 0 & 1 & 1 & 1 & 0 & 0 & 1 & Intestine \\
\hline 24.51 & 967.4173 & 967.3447 & 967.4214 & 188703422 & -1 & 0 & 1 & 1 & 0 & 1 & 0 & 1 & Intestine \\
\hline 24.51 & 1025.4592 & 1025.3904 & 1025.4652 & 603309492 & -1 & 0 & 2 & 2 & 0 & 0 & 0 & 1 & Intestine \\
\hline 25.91 & 1199.5485 & 1199.4576 & 1199.5525 & 109922784 & -1 & 1 & 2 & 2 & 0 & 0 & 0 & 1 & Intestine \\
\hline 35.07 & 1345.6064 & 1345.5127 & 1345.6195 & 191482509 & -1 & 0 & 2 & 2 & 0 & 0 & 1 & 1 & Intestine \\
\hline 29.88 & 1416.6435 & 1416.5386 & 1416.6487 & 72829438 & -1 & 0 & 2 & 2 & 0 & 1 & 0 & 1 & Intestine \\
\hline
\end{tabular}

Note: All $\mathrm{m} / \mathrm{z}$ values including the expected theorectical masses and experiemtnally detected masses refer to the monoisotopic masses. Intensity of each detected component is based on the peak areas of their respective extracted ion chromatograms (XIC). 